\begin{tabular}{ccc}
\hline & International Journal of Engineering \& Technology, $7(2.7)(2018) 968-971$ \\
SPC & International Journal of Engineering \& Technology \\
Website: $w w w . s c i e n c e p u b c o . c o m / i n d e x . p h p / I J E T$ & Research Paper \\
\hline
\end{tabular}

\title{
Heterogeneous networked data recovery from compressive measurements using a copular
}

\author{
Dr. K. Raghavarao ${ }^{1 *}$, M. Vamsi Krishna ${ }^{1}$, P. Sai Chaitanya ${ }^{1}$, E. ShivaKumar ${ }^{1}$ \\ ${ }^{1}$ ECSE Dept. HOD KLEF \\ *Corresponding author E-mail: Raghavarao@kluniversity.in
}

\begin{abstract}
Expansive scale information accumulation by methods for remote sensor system and web of-things innovation postures different difficulties in perspective of the confinements in transmission, calculation, and vitality assets of the related remote gadgets. Com-pressive information gathering in light of packed detecting has been demonstrated an appropriate answer for the issue. Existing plans misuse the spatiotemporal connections among information gathered by a particular detecting methodology. Be that as it may, numerous applications, for example, ecological checking, include gathering heterogeneous information that are inherently corresponded. By this examination, we are trying to propose the use of relationship from different heterogeneous signs while recouping the information from compressive estimations.
\end{abstract}

Keywords: Use about five key words or phrases in alphabetical order, Separated by Semicolon.

\section{Introduction}

In this paper, we are trying to propose a novel recuperation calculation - based upon conviction proliferation standards - that use related data from various heterogeneous signs. To productively catch the factual conditions among various sensor information, the proposed calculation utilizes the measurable model of copula capacities. Analyses with heterogeneous air-contamination sensor estimations demonstrate the proposed configuration gives noteworthy execution upgrades against best in class compressive information social affair and recuperation plots that utilization traditional comsqueezed detecting, packed detecting with side data, and disseminated compacted detecting.

The rising worldview of shrewd urban communities has set off the improvement of new application spaces, for example, environmental checking and keen versatility.

This work demonstrates to viably use the dependencies among differing (nom de plume, heterogeneous) information writes so as to fundamentally decrease information rates in the system. This lessening converts into control reserve funds at the remote hubs or IoT gadgets, which work under somber restrictions in vitality assets. Productive plans should, by the by, misuse intra-and between information conditions on the decoder in order to moderate the computational exertion at the remote sensors and to lessen vitality requesting between sensor correspondence. In addition, with a specific end goal to shield control reserve funds, gadgets ought to convey over little separations through multi-jump remote transmissions, in particular, from neighbor to neighbor, as opposed to straightforwardly to a sink. At last, as data is sent over blunder inclined remote channels, information gathering and recuperation plans ought to accommodate heartiness against correspondence clamor.

\section{Related work}

Related examinations on the issue of information gathering and recov-ery for WSNs proposed to decrease information rates by gettogether focuses with related readings into social occasions or by enabling a little subset of spotlights to transmit information passing on most by a wide margin of the data in the structure.Elective examinations concentrated on general information weight systems including dif-ferential beat code control (DPCM) trailed by entropy encoding . Unmistakable blueprints considered total wavelet change coding or offered an adaptable confirmation between a passed on wavelet change and a spread pre-enunciation based game plan These systems, notwithstanding, require extra between sensor correspondence, developing the transmission of overhead data over the structure.

\section{Contribution}

We propose another heterogeneous engineered information recovery technique, that which builds the likelihood of Bayesian CS with conviction impelling our check progression vances over this idea by merging unmistakable. This is instead of past examinations which consider flag recuperation helped by a solitary side data hail. Past CS approaches depict the reliance among homogeneous sensor readings utilizing the small typical section despite headways demonstrate [24]; basic included substance models or joint Gaussian blend models Dissimilar to these examinations, we show the reliance among heterogeneous information sources utilizing copula limits we look at copula-based graphical models - in light of conviction affecting for information recuperation. Copula limits exhibit the superfluous dispersals and the reliance structure among the information uninhibitedly; everything considered, they get eccentric conditions among gathered information more precisely than existing strategies 
Experimentation utilizing manufactured information and what's more one of a kind air-contamination sensor estimations from the USA Environmental Protection Agency shows that, for a given information rate, the proposed method diminishes the age blunder of the recouped information concerning set up CS with side data, and DCS based procedures. On the other hand, for a given expansion quality, the strategy offers immense rate hypothesis stores, in this manner acknowledging less structure advancement and decreased vitality.

\subsection{Pressed sensing with side data}

CS couple be acclimated can be use to identified with the pennant of intrigue, called side data, which is given from the before to the decoder, to help diversion In CS with side data, the decoder intends to patch up $\mathrm{x}$ from the estimations, the framework An, and a side data vector $\mathrm{w}$ that is related with $\mathrm{s}$. The work is offers assertions to a specific system for arranging side data into CS. Specifically, one adds to the goal of (1) the '1-standard of the separation between the overhaul variable $\mathrm{s}$ and the side data $\mathrm{w}$, yielding the ${ }^{-}-^{-} 1$ minimization issue:

\subsection{Passed on packed sensing}

The sensor signals xj $2 \mathrm{Rn} ; \mathrm{j} 2 \mathrm{fl} ; 2 ;:$; g, are required to have a depiction $x j=(s c+s j)$, where $s c 2 R n$ is a meager part normal to all signs, sj $2 \mathrm{Rn}$ is an insufficient headway section phenomenal to each banner, and $2 \mathrm{Rn} \mathrm{n}$ is the sparsifying premise. Each sensor $\mathrm{j} 2 \mathrm{fl} ; 2$; :; g uninhibitedly encodes the consider signal by expecting it onto a distinguishing structure $\mathrm{j}$ and transmits the low-dimensional estimations $y j=j x j$ to the sink. The sink, along these lines, together reproduces the signs by enlightening:

\subsection{Compressive data gathering for WSNS}

The compressive data gathering approach in holds quick to a multiskip correspondence circumstance in which each center point exchanges a weighted entire of sensor readings to a neighboring center. Specifically, consider an arrangement of $n$ centers and let xi two $\mathrm{R}$ mean a scalar examining of center point as shown in figure

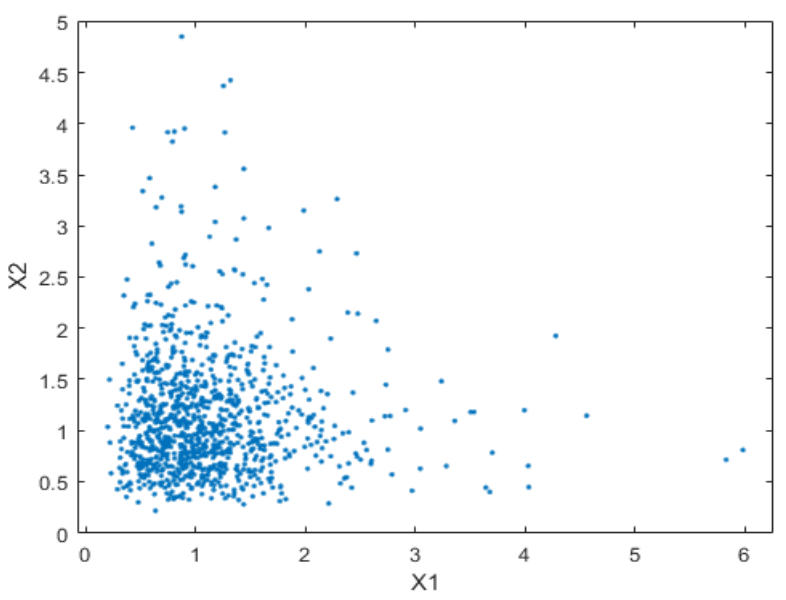

Fig. 1: Shows Compressive Data Gathering.

On the other hand, in the approach of each middle point I transmits with likelihood pi its analyzing plainly to the sink. Along these lines, the sink collects estimations $\mathrm{y}=\mathrm{x}$, where is a to an extraordinary degree pitiful twofold system with one fragment proportionate to 1 for each line and at most one portion indistinguishable to 1 for each segment, while the distinctive parts are zero. The sink by then handles (1) to recuperate the readings from every single one of the middle focuses in the structure.

\section{CS for heterogeneous networked infor- mation}

Best in class compressive information get-together and recuperation courses of action givenan recognizing method. In any case, current WSN and IoT setups consolidate assembled recognizing contraptions gathering heterogeneous information; for example, phenomenal air debasement estimations $(\mathrm{CO}, \mathrm{NO} 2, \mathrm{O} 3, \mathrm{SO} 2)$ are collected in a trademark checking setup. We introduced a design that regularly rehashes heterogeneous related information to compressive estimations, by utilizing both intra-and between source information conditions.

The social event of little vectors fs(l)gl $=1$ utilizing the outfit of The information gathering outlines that were estimations vectors fy(l)gl ${ }^{`}=1$ and the lattices $\mathrm{f}(\mathrm{l}) \mathrm{gl}{ }^{`}=1$.

Happens, DCS gets together of heterogeneous information. we expect that heterogeneous information, for example, exceptional air hurts, which have the correspondence plan is a line diagram, beginning at center point particular veritable properties. 1 and conclusion at focus point $n$. Focus $n$, hence, is connected with the The strategy we propose, then again, use shifting sink focus point. The estimations of information create 1 are aggregated and related developments through copula limits Copula transmitted as was delineated in Section II-D: focus point 1 measures limits, cleared up in detail in Section IV-An, are parts $\mathrm{x} 1(1)$, and transmits $1(1 ;) 1 \times 1(1)$ to focus 2 , where $1(1 ;) 1$ is randomly of a quantifiable structure to successfully get conditions delivered; frameworks have just few non-zero areas, which are either 1 or 1 , with break even with the underlying speculation with likelihood. As appeared in, they can incite correct what's all the more, smart conviction affecting based CS disentangling, as constrained to thick Gaussian frameworks

Regardless, as appeared in our trial happens, DCS does not valuably get the covered conditions among heterogeneous information, for example, remarkable air contaminations, which have arranged honest to goodness properties.

we are utilize copula capacities to encourage picking up from other information makes in the increase out of a given information make or, in different words, as an approach to manage compose distinctive side data.

Our examinations in Section VI display that it is unquestionably in light of the way that it utilizes different side data signals at the recovery coordinate that our game plan beats the best in class frameworks

\section{Copula-based belief propagation}

All know that delineate our excitement figuring, executed on the sink focus. As showed, the insufficient vectors are copied successively: in any case, s (1), by then s (2), et cetera. The diversion of each s (1) along these lines utilizes the individual estimations y (1), and furthermore the starting at now rehashed information composes $\mathrm{s} ; \mathrm{s}$ as side data.

We get the course of action of Bayesian CS as it routinely handles our joint genuine portrayal of the related modalities. We begin by figuring the back allotment of the subjective vector S (1), tending to the scantyvectors of coefficients of information make 1, given the diverse estimations $Y(1)$ and the essential 11 information makes: The factor focus focuses are related to the broadly engaging components in the articulation, for this situation Factor plot relating to the back assignment the variable concentrations are tended to with circles and the factor centers with squares. A message from variable concentration point $\mathrm{s}$ (il) to factor center $\mathrm{fZ}$ at accentuation is proposed with qi! $Z$, and a message in the turn heading is deduced with rZ!i.

Concerning association with the farthest right consider focus focuses prescribes that each term fSi (l) jSi (1) Si (1 1) a be granted as the irrelevant pdf $\mathrm{f}$ (1) times a cor-Si rection term that gets data from the starting at now changed information forms. Undoubtedly, expecting we approach measures $b s(k)$ of $s(k)$, for $k<1$, there holds 


\section{Quantifiable displaying using copulas}

We now depict how to show to a great degree heterogeneous data using copula limits. Allow $\mathrm{S}(1)$ to mean the sporadic vari-related with the breaking down of source There exist a few bivariate and multivariate copula fam-ilies routinely sorted out into obvious what's progressively, unequivocal. Certain copulas have densities with no focal close shape elucidation, however are gotten from without a doubt got a handle on distribu-tions.

\section{Examinations}

We survey the data recovery execution of the proposed copulabased blueprint using built data (cf. Section VI-An) and also honest to goodness sensor readings taken from the air pollu-tion database of the US Environmental Protection Agency (EPA) [(cf. Fragment VI-B). In addition, in Section VI-C, we consider the impact of the proposed system on the essentialness use of the remote devices.

\subsection{Results on engineered data}

Keeping in mind the end goal to assess the proposed copula-based method, we imitate the approach portrayed. In figure 2

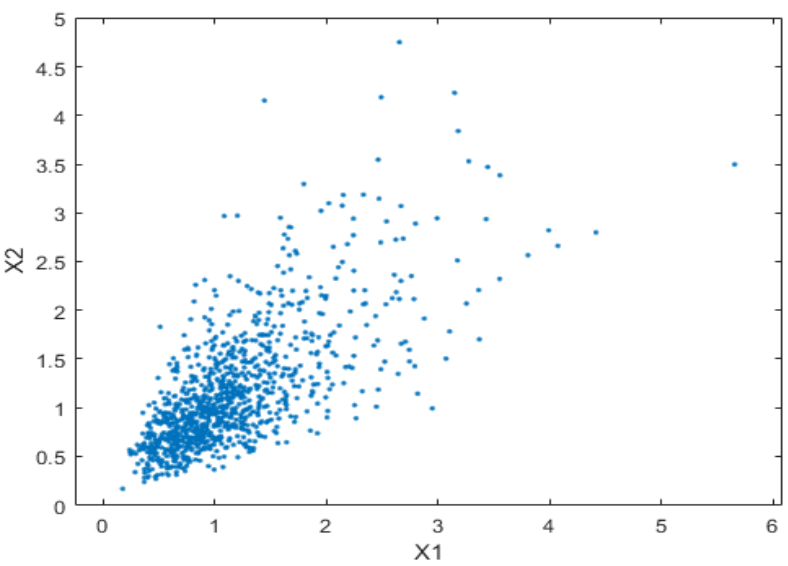

Fig .2: Shows Quantifiable Displaying

We consider the vectorized readings $\mathrm{x}$ (1) x (2) of two extremely subordinate information creates aggregated at a given time occasion by a WSN and their compressible portrayals s (1) s (2) out of a prelude. Following existing stochastic models for the season of spatially-related WSN information, we expect that both $\mathrm{x}$ (1) and $\mathrm{x}$ (2) are Gaussian. We in addition recognize that $\mathrm{x}(1)$ is stationary (its instability is unsurprising transversely completed readings), while $\mathrm{x}$ (2) is piece-wise stationary (its change moves crosswise over completed social events of readings). Taking as the DCT introduce, it can be genuinely recognized that the coefficients in s(1) are Gaussian, however the coefficients in $\mathrm{s}(2)$ take after the Laplace dispersal with probability $\rho=0.1$ to 0.8 as shown fig
Laplace Dispersal with Probability 0.1 and 0.8
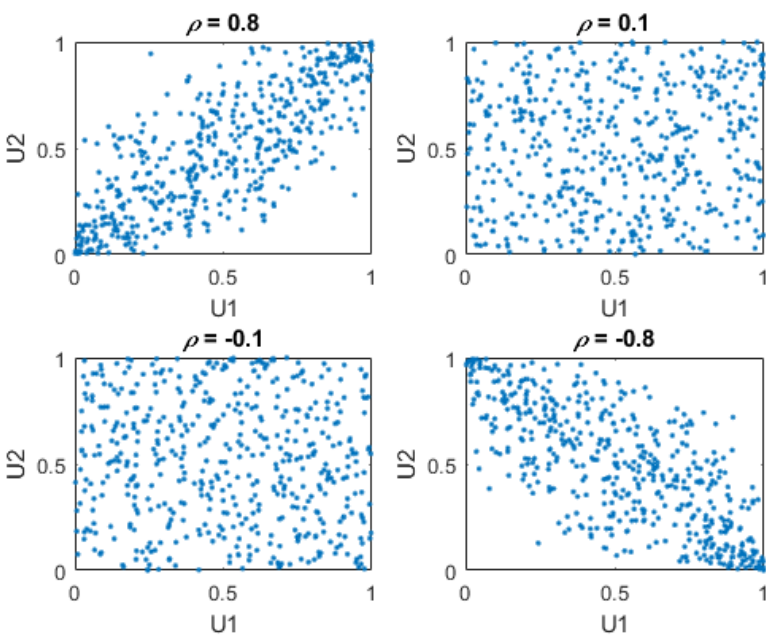

\subsection{Results on real air contamination data}

The Air Quality System database of EPA aggregates air quality estimations taken by more than 4000 watching stations, which gather hourly or reliably measurements of the merging of six poisons

Sparsifying Basis Selection: We at first perceived a not too bad sparsifying purpose behind the data. Following the framework as shown in figure

Here the simulation frame work is for 1000 wsn samples with different frequencies with $(0,1)$ random values

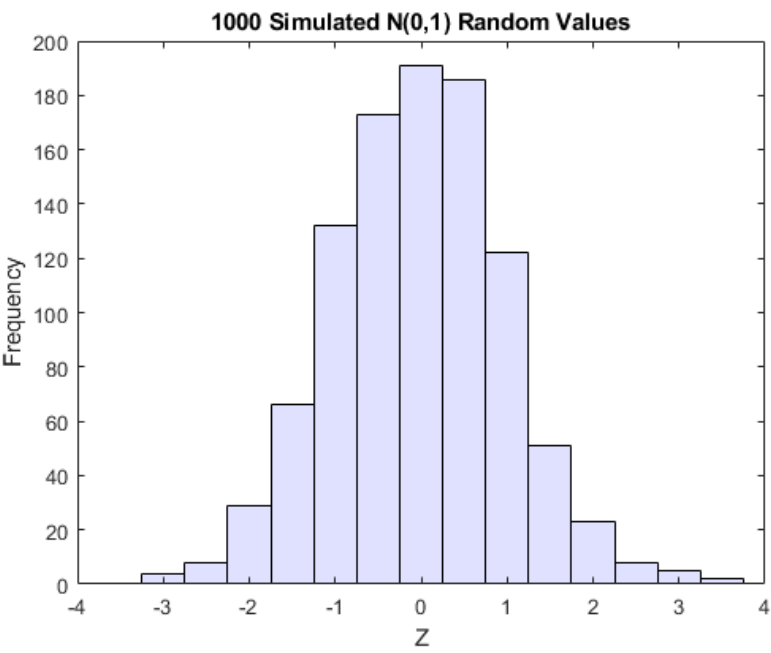

Fig. 3: With Transformation of Unit Function.

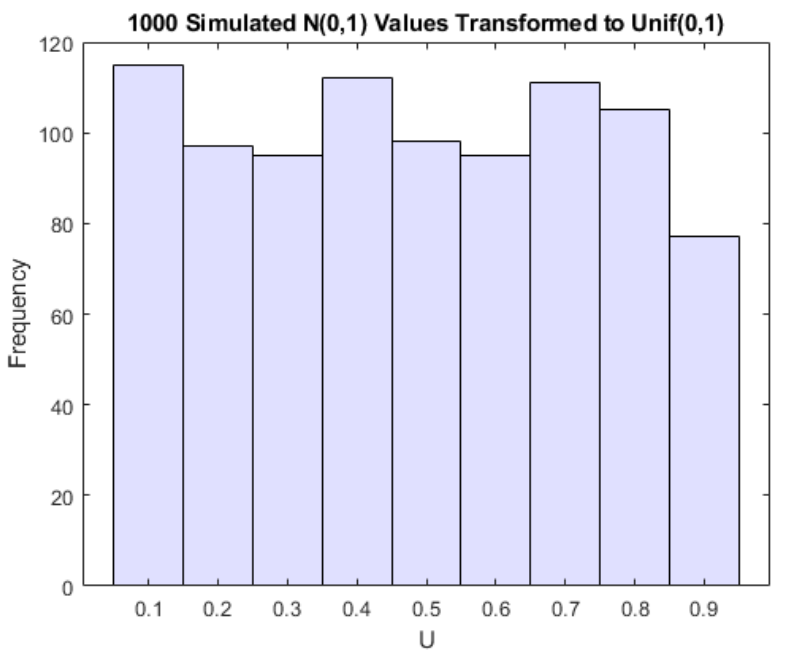

Fig. 4: With Values Transformed to Gamma $(2,1)$ with Bivariate Distribution. 


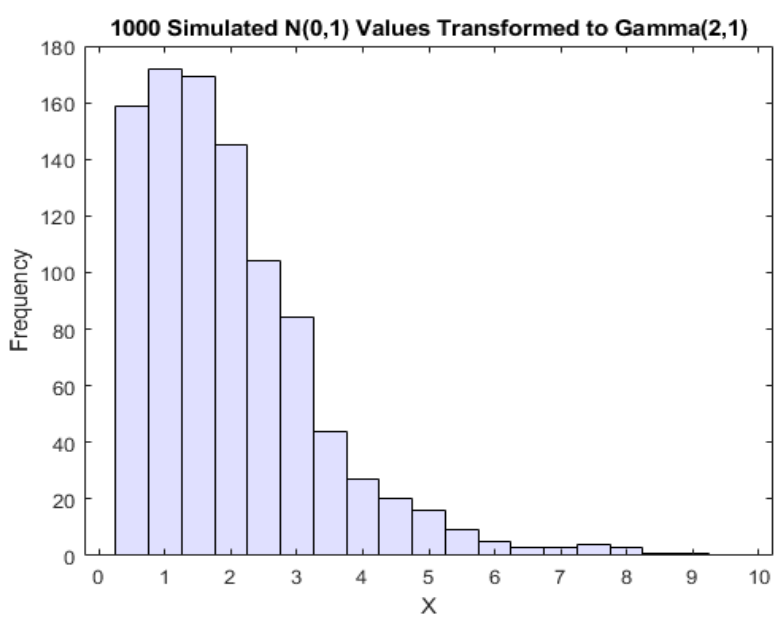

Fig. 5:

Configuration Depicted In The Past Area, We Dealt With The Readiness Data Into Bits Of N Readings Per Harm. Remembering the True Objective to Outline A Square

$\mathrm{X}$ (1), readings must have the same timestamp and be assessed by neighboring stations, holding quick to the LoRa transmission isolate criteria.

\section{Conclusion and future work}

We looked out for the issue of information recuperation from compressive estimations in clearing scale WSN applications, for example, air-contamination viewing. To satisfactorily get quantifiable conditions among heterogeneous sensor information, we utilized copula confines This empowered us to devise a novel CS-based delight check, in light of conviction spread which use diverse heterogeneous signals (e.g., air contaminations) as side data in orderto enhance evolving. Examinations utilizing constructed information and true blue sensor information from the USA EPA demonstrated that the proposed conspire fundamentally updates the possibility of information multiplication worried earlier best in class systems even under recognizing and correspondence clamor. Also, we exhibited that, for a given data redoing quality, the proposed scheme offers low encoding multifaceted design and lessened radio transmissions appeared differently in relation to the best in class, thus provoking essentialness venture stores at the remote devices. We assume that our arrangement reasonably deals with the solicitations of a far reaching scale watching application. Future work should center around assessing the system on elective datasets, for instance, the Intel-Berkeley Lab dataset the dataset from the Center for Climatic Research, and the indoor dataset

\section{References}

[1] J. Inglada and A. Giros, "On the real capabilities of remote sensing for disaster management-Feedback from real cases," in Proc. IEEE IGARSS, Sep. 22-24, 2004, vol. 2, pp. 1110-1112.

[2] A. Singh, "Digital change detection techniques using remotelysensed data," Int. J. Remote Sens., vol. 10, no. 6, pp. 989-1003, 1989.

[3] C. Oliver and S. Quegan, Understanding Synthetic Aperture Radar Images. Norwood, MA: Artech House, 1998.

[4] F. Melgani, G. Moser, and B. Serpico, "Unsupervised change-detection methods for remote-sensing images," Opt. Eng., vol. 41, no. 12, pp. 3288- 3297, Dec. 2002.

[5] Y. Bazi, L. Bruzzone, and F. Melgani, "An unsupervised approach based on the generalized Gaussian model to automatic change detection in multitemporal SAR images," IEEE Trans. Geosci. Remote Sens., vol. 43, no. 4, pp. 874-887, Apr. 2005.

[6] G. Moser and S. B. Serpico, "Generalized minimum-error thresholding for unsupervised change detection from SAR amplitude imagery," IEEE Trans. Geosci. Remote Sens., vol. 44, no. 10, pp. 2972 2982, Oct. 2006
[7] L. Bruzzone and D. F. Prieto, "Automatic analysis of the difference image for unsupervised change detection," IEEE Trans. Geosci. Remote Sens., vol. 38, no. 3, pp. 1171-1182, May 2000

[8] K. Conradsen, A. A. Nielsen, J. Schou, and H. Skriver, "A test statistic in the complex Wishart distribution and its application to change detection in polarimetric SAR data," IEEE Trans. Geosci. Remote Sens., vol. 41, no. 1, pp. 4-19, Jan. 2003.

[9] G. Moser, S. B. Serpico, and G. Vernazza, "Unsupervised change detection from multichannel SAR images," IEEE Geosci. Remote Sens. Lett. vol. 4, no. 2, pp. 278-282, Apr. 2007.

[10] J. Inglada and G. Mercier, "A new statistical similarity measure for change detection in multitemporal SAR images and its extension to multiscale change analysis," IEEE Trans. Geosci. Remote Sens., vol. 45, no. 5, pp. 1432-1446, May 2007.

[11] E. J. M. Rignot and J. J. van Zyl, "Change detection techniques for ERS-1 SAR data," IEEE Trans. Geosci. Remote Sens., vol. 31, no. 4, pp. 896-906, Jul. 1993

[12] .R. Touzi, A. Lopès, and P. Bousquet, "A statistical and geometrical edge detector for SAR images," IEEE Trans. Geosci. Remote Sens., vol. 26, no. 6, pp. 764-773, Nov. 1988.

[13] F. Bujor, E. Trouvé, E. Valet, J. Nicolas, and J. Rudant, “Application of log-cumulants to the detection of spatiotemporal discontinuities in multitemporal SAR images," IEEE Trans. Geosci. Remote Sens., vol. 42, no. 10, pp. 2073-2084, Oct. 2004.

[14] V. Alberga, M. Idrissa, V. Lacroix, and J. Inglada, "Comparison of similarity measures of multi-sensor images for change detection applications," in Proc. IEEE IGARSS, Barcelona, Spain, Jul. 23-27, 2007, pp. 2358-2361.

[15] J. Inglada and G. Mercier, "The multiscale change profile: A statistical similarity measure for change detection in multitemporal SAR images," in Proc. IGARSS, 2006, pp. 212-215.

[16] M. Basseville and I. Nikiforov, Detection of Abrupt Changes: Theory and Application. Englewood Cliffs, NJ: Prentice-Hall, 1993.

[17] M. G. Kendall, the Advanced Theory of Statistics, vol. II. London, U.K.: Griffin, 1945.

[18] J. Inglada, J.-C.Favard, H. Yesou, S. Clandillon, and C. Bestault, "Lava flow mapping during the Nyiragongo January, 2002 eruption over the city of Goma (D.R. Congo) in the frame of the international charter space and major disasters," in Proc. IGARSS, Jul. 21-25, 2003, vol. 3, pp. 1540-1542.

[19] M. Buchinsky, "Recent advances in quantile regression models: A practical guideline for empirical research," J. Hum. Resour., vol. 33, no. 1 , pp. 88-126, 1998.

[20] R. Koenker and J. Gilbert Bassett, "Regression quantiles," Econometrica, vol. 46, no. 1, pp. 33-50, Jan. 1978. 